\title{
Deployment Adviser tool for Wireless Sensor Networks
}

\author{
Amarlingam M, Adithyan I, P Rajalakshmi \\ Department of Electrical Engineering \\ Indian Institute of Technology Hyderabad \\ Hyderabad, India \\ Email: ee13p1003, ee09b001, raji@iith.ac.in
}

\author{
Yasutaka Nishimura,Masaya Yoshida, Kiyohito Yoshihara \\ KDDI R\&D Laboratories Inc. \\ Green and M2M Application Laboratory \\ Iidabashi, Japan \\ Email: yu-nishimura,my-yoshida, yosshy@kddilabs.jp
}

\begin{abstract}
This paper presents a system for the purpose of field deployment of nodes in wireless sensor networks. We propose a mobile phone based deployment adviser tool which is robust as well as practically implementable. The tool advises a layman deployer to create a optimized wireless sensor network by placing of the nodes according to application requirements. The tool is presented here as logically linked sub-modules. Each sub-modules are described in detail. Also we propose an algorithm which helps in distributing the power consumption among the nodes in the network, thus, increasing the network lifetime. The adviser tool has been verified by implementing it in IITH mote. The tool we propose has significant implication since it greatly eases, but more importantly extracts the best performance possible while deploying the wireless sensor networks.
\end{abstract}

Index Terms-Routing, Link Quality, Network Topology

\section{INTRODUCTION}

Wireless sensor network's (WSN) are forecasted to see tremendous growth in the coming years. The focus of research on this emerging technology has been to satisfy the functional requirements while taking into consideration the resource constraints. Typically, in WSN setting, there is a Base station (B.S), Sensor nodes (S.N) and Relay nodes (R.N). Often location of these sensor nodes is predetermined, the positions are dependent on the nature of the application. For instance, one would like to measure the temperature in a particular room and measure the $\mathrm{CO}_{2}$ level in other. And there is a B.S sitting far away from these sensor nodes collecting all the data from the sensors. The B.S transmits back relevant commands to these sensor nodes if required. R.N provides the path from the sensor nodes to the base station and vice-versa. The location of all these nodes have to be estimated carefully to achieve the best performance, but often the question of how to deploy the nodes so that they ensure connectivity and at the same time optimize the performance is a very tough problem and has been shown to be NP-Hard [1-3]. The way to overcome this problem is to propose a heuristic design and to find suboptimal solutions.

As mentioned earlier few theoretical work exist which discuss on the optimal solutions for relay node placement. In [4] the authors discuss on how to find the minimum number of R.N's and where they can be placed in order to meet the constraints of network lifetime and connectivity, [5] tries to address a similar problem. [6] discusses deployment of nodes according to traffic pattern and [7] talks about redeployment schemes. [8-10] is a series of extended work on deterministic placement of relay nodes. The focus of these theoretical works is never on building a unified deployment advisor system. Such slight overlook may cost dearly in terms of performance. These schemes are too theoretical to use in a real world setting. They assume ideal scenarios and produce results. Accurate placement of nodes at these estimated location is difficult, often due to poor location information. In real environment it is very difficult to replicate the results obtained in simulation because the simulations can't model the environmental obstacles brought into the channel. Due to which the predicted locations from the simulation perform poorly when replicated in real time. Authors of [16] discussed about existing deployment methods like perceptual method, energy consumption model and coverage ratio model for sensor networks. In [17] authors proposed smartphone based tool that track sensor locations and provides debugging tools.

In this paper we propose a deployment advisor tool which takes into account all previously mentioned challenges. As part of the advisor tool, we also propose an intelligent algorithm which help in ensuring the power is consumed equally among all the nodes in the network, thus increasing the network lifetime. It has been field tested by implementing on IITH Mote(a mote developed in Indian Institute of Technology, Hyderabad) to ensure that they indeed work. The rest of the paper is organised as follows. In Section II we will introduce our advisor scheme and break down it down into various sub modules. Each of the modules have a dedicated task and they proceed in a chronological fashion. In the following sections III and IV we will be explaining each of these sub modules in more detail. In Section $\mathrm{V}$, we discuss a algorithm for balancing out the power consumption across different nodes in the network. Section VI presents the experimental results. Section VII concludes the paper.

\section{ADVISOR SCHEME}

We restate the problem here. One is given the location of sensor nodes, which is application specific, and there exists a base station which collects the information from these nodes. The challenge is the deterministic placement of relay nodes that ensures robust connectivity and obtains optimal network 


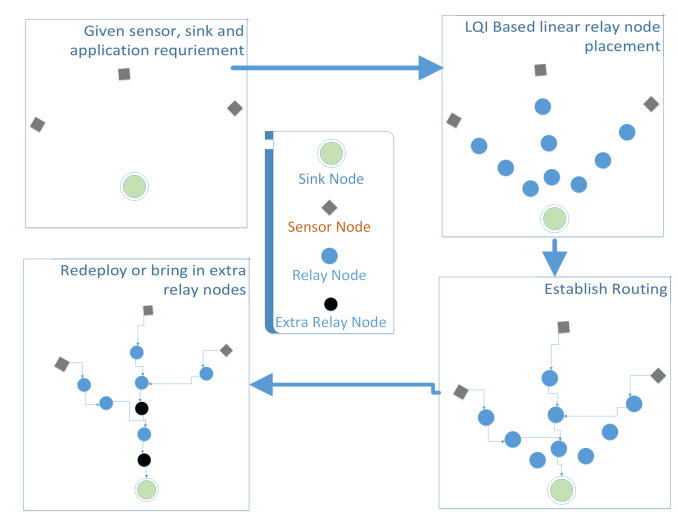

Fig. 1: Sub-Modules involved in the Advisor scheme

lifetime. In the terminology of [11], our strategy is a static node placement technique. Our deployment methodology is controlled with an optimization objective of network longevity, connectivity and the role played by the node in WSN is relay node.

Our deployment proceeds through various logically well connected stages. In brief, the model has the following submodules in it:

- Placement of Relay nodes according to link quality.

- Establishing a Routing layer.

- Redeploy as directed by the advisor.

This is depicted in figure 1.

\section{Placement of Relay nOdes according to Link QUALITY}

The location of sensor and B.S is fixed. Now the human deployer, carrying a R.N and mobile phone in hand, starts from the B.S and moves towards the sensor nodes. He constantly monitors the link quality (LQI), which is displayed in the mobile phone and just when the LQI crosses a certain configurable threshold he places the relay node there. He keeps repeating the process until he reaches the sensor node. This is a simple Linear Link quality based deployment.

At the same time in the background the mobile phone periodically keeps collecting the data of Link-quality vs Distance. The distance is computed with the help of the localization technique. This reference table is stored in the mobile. Table is explained later. A small hint is that we earlier mentioned how certain strategies in the literature are limited by their assumption of environmental disturbances. In our case there are no assumptions, the environment is characterised in real time. And it is discreetly embedded in deployment process itself. One need not spend time and money to characterize the environmental obstacles. This is process depicted in the figure 2 .

\section{Route IdentificAtion AND Network Lifetime COMPUTATION}

One could easily see that by employing a routing layer one could do away with some of the relay nodes. A routing protocol would use only some of the relay nodes. Hence the unused

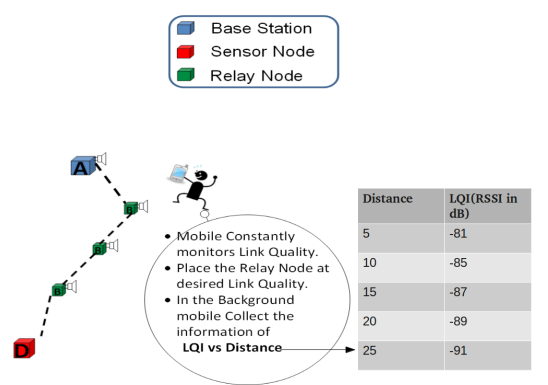

Fig. 2: Linear Link quality based deployment along with LQI vs Distance data collection

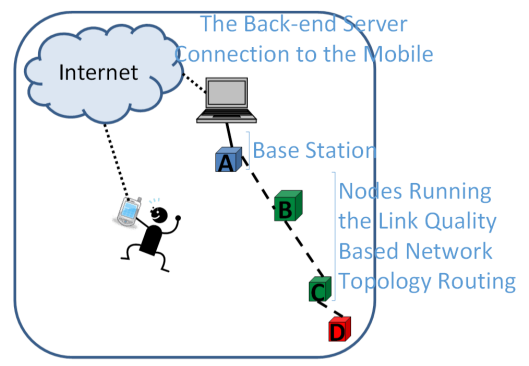

Fig. 3: Connection between the Mobile and the Base Station through the server

relay nodes are redundant and could be discarded. Hierarchical routing is often used to maintain the energy consumption by involving them in multihop communication. There are various hierarchical routing protocols such as LEACH [12], PEGASIS [13], TEEN [14] among others. In our scheme we use similar to Hierarchical-PEGASIS [15] routing protocol. In brief the routing protocol implemented in the IITH mote goes through the following stages:

1) Hierarchy : Hierarchy established among nodes depending upon their distance from B.S.

2) Next neighbour : Each selected node in a particular level picks a particular node in the upper level of hierarchy according to the link quality.

3) Communicate to B.S : All Nodes communicate Information like Heirarchy level, Traffic, Residual energy, and next neighbour among others to B.S.

4) Data Transmission : Each selected node transmits data to the node in the upper level of the hierarchy.

After the step 3, the base station forwards the information to the mobile phone through the back-end server connection. Now the mobile creates the network topology. This is depicted in figure 3. And with the additional information it has gathered it simulates the network and calculates the network lifetime. This is the original network lifetime(N.L). This N.L will be compared with the N.L obtained after we redeploy the nodes. This will give a sense how better one's redeployment algorithm is working. 


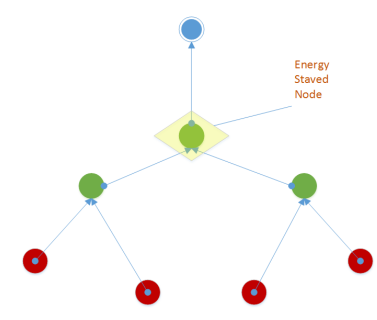

Fig. 4: Showing a typical scenario which involves a Energy Starved Node

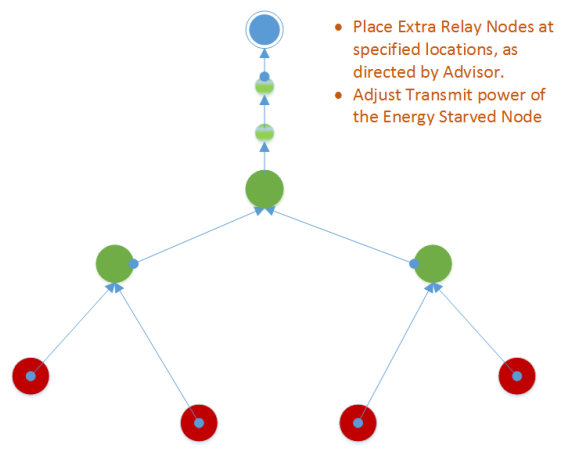

Fig. 5: Placing Extra relay around the Energy Starved Node to improve Network Lifetime

\section{INTELLIGENT ALGORITHM AND REDEPLOYMENT OF NODES}

Even though the above mentioned routing protocol ensures a route between a sensor node and a B.S, it is not effective in terms of network lifetime. Certain nodes are utilized heavily and they are soon exhausted. Figure 4 shows one such typical scenario in WSN. So we propose the following novel method to balance the energy consumption.

By deploying the extra relay nodes around the energy starved nodes, one could also balance the energy consumption. In effect what we are doing is bringing our next neighbour close to us. The above method is shown in figure 5 .

Now the question is given a Network Topology, how do we design an optimum algorithm such that energy consumption across all the nodes is balanced. As explained earlier, the answer depends upon the path we take. We take up the simpler problem of placing extra relay nodes around energy starved nodes. We have developed an algorithm which would take in parameters and give out how many relay nodes should be place around an energy starved node and where to place them.

\section{A. Parameters required by the algorithm}

- Network Topology.

- LQI vs Distance Range (TD) for every possible Transmit power.

- Sampling Rate and Payload information for every node.

Network topology is the current orientation of the network. That is available from the module-2. The LQI vs Distance table for every possible transmit power is required to judge by how much does a particular link weaken it terms of link

\begin{tabular}{|l|l|}
\hline Variable & Description \\
\hline$N$ & $\begin{array}{l}\text { An array whose length is equal to number of } \\
\text { nodes in network }\end{array}$ \\
\hline$M_{e}$ & Node ID of the most energy starved node \\
\hline$L_{e}$ & Node ID of the least energy starved node \\
\hline$N_{t i}^{\prime}$ & Transmission Index array \\
\hline$N_{t i}$ & Normalized Transmission Index array \\
\hline$N_{s}$ & Sampling rate \\
\hline$N_{s b}$ & $\begin{array}{l}\text { Array holding the sub-nodes attached with a } \\
\text { particular node }\end{array}$ \\
\hline$T X_{\text {old }}$ & Array holding new transmit power \\
\hline$T X_{n e w}$ & Array holding old transmit power \\
\hline$r_{l}$ & $\begin{array}{l}\text { Array holding the number of relay nodes between } \\
\text { the node and its next neighbour }\end{array}$ \\
\hline$d_{l}$ & $\begin{array}{l}\text { Array holding the distance at which the new relay } \\
\text { nodes have to be placed }\end{array}$ \\
\hline
\end{tabular}

Fig. 7: Variable used in the algorithm

quality when we reduce the transmit power. Number of relay nodes to be brought is decided by degree of link quality reduction. Sampling rate and the payload information are required to arrive at a normalized data handled for every node. The algorithm is depicted pictorially in a simplified form for the purpose of understanding. It is depicted in figure 6 .

\section{B. Algorithm}

The variables used in the algorithm are depicted in ??. All the array variables are by default of the length equal to number of nodes in network, unless specified. The information in a particular index of the array corresponds to that node whose ID $=$ Index. For example $T X_{n e w}[3]$ is the new transmit power of node 3 .

We define a variable named transmission index. From the network topology and sampling rate acquired we could assign two identifiers to every node :

- Sampling rate : $N_{s}$

- Sub-nodes attached : $N_{s b}$

Using this we find out the value of transmission index, $N_{t i}$ of every node as:

$$
\begin{aligned}
& N_{t i}^{\prime}=N_{s}+\sum_{i=\text { Over all } N_{s b}} N_{t i}^{\prime}(i) \\
& N_{t i}=\text { Normalized }\left\{N_{t i}^{\prime}\right\}
\end{aligned}
$$

The $N_{t i}^{\prime}$ is computed from down to top, i.e starting from least hierarchy level. Once every node in least hierarchy is done we move to the next highest hierarchy. Typically $N_{s}$ for a relay node is 0 . For the purpose of understanding the values of $N_{t i}$ is computed for a simple case and is depicted in figure 8 . The ones shaded in blue correspond to relay nodes and assume every sensor has a sampling rate of 1 . Hence their $N_{s}=1$. Relay nodes have a $N_{s}=0$. Using this we can compute the $N_{t i}^{\prime}$ for all other nodes in the network.

$N_{t i}^{\prime}$ gives the number of packets a particular nodes has to transmit/forward in a second. When we normalize, it gives 


\begin{tabular}{|c|c|c|c|c|c|c|c|c|}
\hline TXPower & 5 & 10 & 15 & 20 & 25 & 30 & 35 & 40 \\
\hline-7 & -83.48 & -89.218 & -92.139 & -94.197 & -95.477 & -95.901 & -96.347 & -96.181 \\
\hline-5 & -81.332 & $(-87.532$ & -90.436 & -93.11 & -94.54 & -95.381 & -95.739 & -96.334 \\
\hline-3 & -79.489 & -84.905 & -88.789 & -91.396 & -92.496 & -94.266 & -94.872 & -95.472 \\
\hline-1 & -77.619 & -83.768 & -87.449 & -89.789 & -91.303 & -92.924 & -93.703 & -94.456 \\
\hline 1 & -75.475 & -81.723 & -85.373 & -87.956 & $-89,435$ & -91.255 & -92.631 & -93.212 \\
\hline 3 & -73.664 & -79.096 & -83.057 & -86.163 & -88.235 & $-(-88.941)$ & -90.612 & -91.354 \\
\hline
\end{tabular}

- Initially Every Node transmits at $3 \mathrm{dBm}$.

- With the received LQI, one could estimated the current distance of the sending node.

- Compute new TX power such that energy consumption is balanced.

- For a given TX power and new LQI required (generally same), one could compute the new range.

- With that compute number of relay nodes required and position of them.

$\begin{array}{ll}\begin{array}{ll}\text { Example: } \\ \text { Current LQI }\end{array} & =-88 . \\ \begin{array}{l}\text { Refer Table }=> \\ \quad \text { Current Distance }\end{array} & =30 \\ \text { New TX Power } & =-5 \\ \text { Required LQI } & =-88 \text { (Gen. Same) } \\ \text { New reduced range } & =10 \\ & \\ \text { No. of Relay required } & =2 \\ \text { Position => Equidistant at } 10 \mathrm{~m} \text { apart }\end{array}$

Fig. 6: Simplified pictorial representation of algorithm to place extra relay nodes around energy starved nodes.

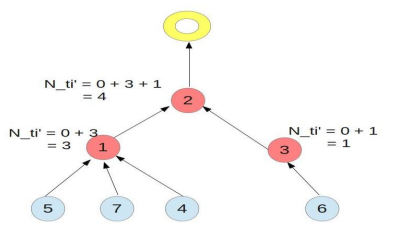

Fig. 8: An example of $N_{t i}$ computation

a relative estimate how much load a particular node handles compared to other nodes. We sort out the most energy starved node to least energy starved nodes, with the help of $N_{t i}$. Once the node are sorted from $M_{e}$ to $L_{e}$, it is stored in $N$ array.

The algorithm is enumerated in the following steps.

1) Access Topology and other relevant information.

2) Compute $N_{t i}$.

3) Store the sorted nodes id's staring from $M_{e}$ to $L_{e}$ in $N$.

4) Initialize $i=1$

5) Initialize $E_{s}=N(i)$

6) $n_{l}=N_{t i}\left(E_{s}\right)$

7) $T X_{\text {new }}\left(E_{s}\right)=\frac{T X_{\text {old }}\left(E_{s}\right)}{n_{l}}$

8) Refer TD, estimate $r_{l}$ and $d_{l}$. Then,

$$
\begin{aligned}
& r_{l}\left(E_{s}\right)=r_{l} \\
& d_{l}\left(E_{s}\right)=d_{l}
\end{aligned}
$$

9) $i++$

10) Repeat steps 5 to 9 until $i=\operatorname{length}(\mathrm{N})$.

$$
\text { STEP - } 6
$$

Out of all the steps in the previous algorithm, step- 6 is the most crucial one. We would take up an example to elucidate it better. Assume node-1 has $N_{t i}(1)=4$ assigned to it. Hence to balance out we need to caluclate new transmit power from old trnsmit power as follow

$$
T X_{\text {new }}(1)=\frac{T X_{\text {old }}(1)}{4}
$$

which is equivalent to a $6 \mathrm{~dB}$ decrease in the $\mathrm{dB}$ power scale. Then

$$
\begin{aligned}
T X_{\text {old }}(1) & =3 d B \\
T X_{\text {new }}(1) & =-3 d B .
\end{aligned}
$$

Assume that the initial distance between Node-1 and its next neighbour is $25 \mathrm{~m}$. Now we refer to the Transmit power vs Distance table as the one shown in figurer 9. By reducing the TX power to $-3 \mathrm{dBm}$ our range has been decreased to $10 \mathrm{~m}$. Hence we would require 1 extra relay node in between node-1 and its next neighbour to establish connection. Hence,

$$
\begin{aligned}
r_{l}(1) & =1 \\
d_{l}(1) & =\text { Placed at } 12.5 \mathrm{~m} \text { away from node- } 1 .
\end{aligned}
$$

\begin{tabular}{|c|c|}
\hline TX(in dBm) & Distance \\
\hline $\mathbf{- 3}$ & $\mathbf{1 5}$ \\
\hline-1 & 20 \\
\hline 1 & 25 \\
\hline $\mathbf{3}$ & $\mathbf{3 0}$ \\
\hline 5 & 40 \\
\hline
\end{tabular}

Fig. 9: Transmit power (vs) Distance table for a particular LQI

The step- 6 is the crucial step in the algorithm. It is meant to draw equal power from all the nodes. 


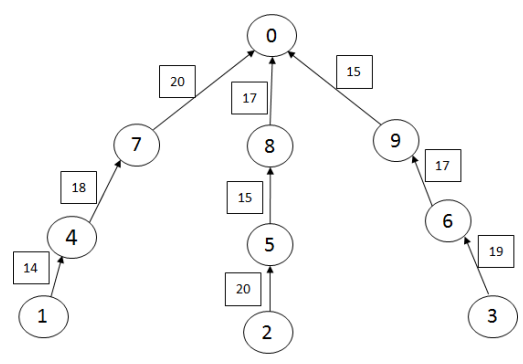

Fig. 10: Network Topology after lay man places relay nodes according to LQI with help of Advisor tool

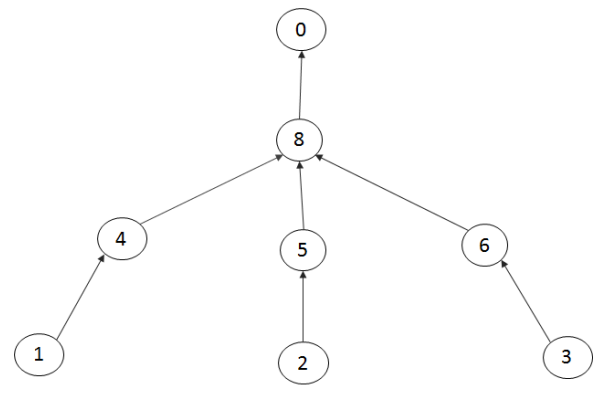

Fig. 11: Topology after routing

\section{Vi. Experimental Results of Proposed Algorithm}

Here we show results of the algorithm for a specific case. Figure 10 shows the network topology after layman deploy nodes moving away from sensor node to base station. Figure11 shows network architecture after routing is done. Here the power consumption is unbalanced. Figure 12 shows network after giving advise, with uniform power consumption for energy starve node.

Information for reading network topology is given below:

$\begin{array}{ll}\text { Node } & \text { Indication } \\ 0 & \text { Base Station } \\ 4,5,6,7,8,9 & \text { Senses and forwards Data (Relay+sensor Node) } \\ 1,2,3 & \text { Senses and send Data(Sensor Node) } \\ 8 & \text { Energy starved node }\end{array}$

We can observe two redundant nodes after routing. These redundant nodes are removed as shown in Figure11. Five extra relay nodes is brought into the network as shown in Figure12 to increase network life time. These extra relay nodes serve the purpose of the balancing out the power consumption across nodes. Thus increasing the network lifetime.

The above conceptualized algorithms have been tested by implementing them On the IITH Mote. The simulation environment was TinyOS. The coding language used is tabulated as below

\begin{tabular}{|l|l|}
\hline nesC & TinyOS Scripting \\
\hline C++ & Part of TinyOS \\
\hline Java & For GUI \\
\hline Android & Application created in the Mobile \\
\hline
\end{tabular}

The linear link quality based deployment, discussed in Step1 , has been implemented on IITH Mote. This is implimented

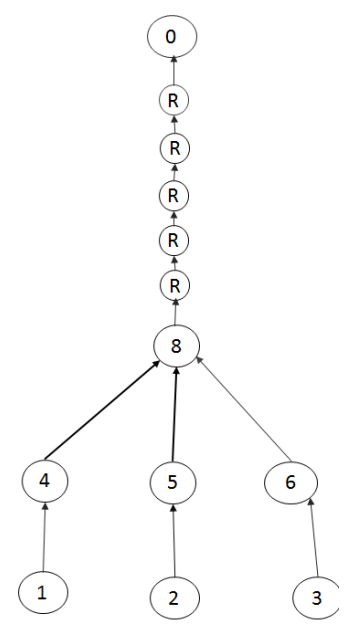

Fig. 12: Topology after giving advise

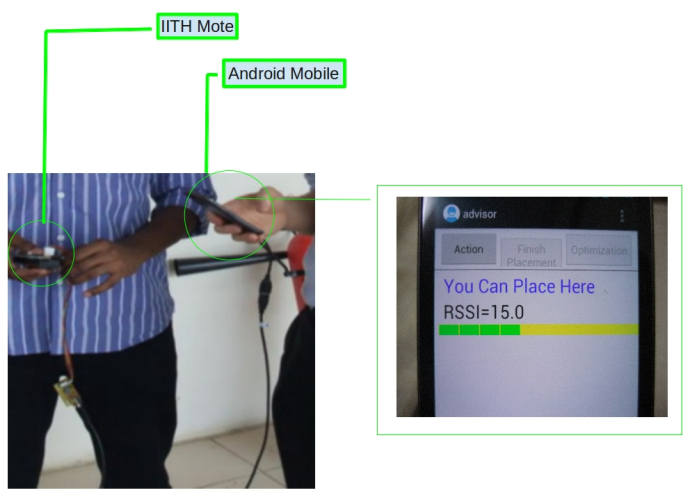

Fig. 13: Picture showing the interface of IITH Mote with a mobile phone and Android application which displays advice.

in two steps as below

- Interface of IITH Mote with the Mobile.

- Android application which advises where to place to a layman.

This is shown in Figure 13. The Network topology protocol has been implemented and verified. A back-end server has been created which accesses the information from the B.S and sends to the Mobile. An Android application takes this information and displays the Network Topology. Also it simulates the network with the help of traffic and estimates the original network lifetime. Both the topology and Network lifetime is displayed by the android APP. Figure 14 shows the the mobile phone displaying topology and the network lifetime. We designed an algorithm which estimates the number and location of relay nodes to be deployed around the energy starved Node.

\section{A. Strengths of Advisor Tool}

- Advisor Tool can detect redundant nodes. from Figure11 we can observe that here 2 relay nodes are found to be redundant nodes 


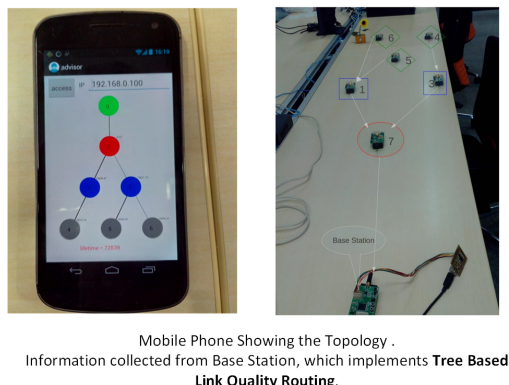

Fig. 14: Mobile accesses information of Network, which is running the algorithm discussed in Section-IV, from B.S. Server acts at the link b/w mobile and B.S.

- Advisor Tool can support the construction of stable sensor network.

- Advisor Tool can prolong the network lifetime.

We observed network life time before giving advise and after advise. We got network life time before giving advise $\mathbf{N l}=\mathbf{3 5 . 5 4 2 2} \mathrm{hrs}$, network lifetime after advise $\mathrm{Nl}=40.39$ hrs

\section{CONCLUSION}

Advisor tool is proposed to create an optimized Wireless Sensor Network for placing the nodes according to application requirements. Algorithm which helps in distributing the power consumption among the nodes in the network is also developed and tested in real time field experiments. It has been observed from the results that algorithm proposed increases the network lifetime. The adviser tool has been verified in field tests with IITH mote.

\section{REFERENCES}

[1] Efrat, Alon, Sariel Har-Peled, and Joseph SB Mitchell. "Approximation algorithms for two optimal location problems in sensor networks," Broadband Networks, 2005. BroadNets 2005. 2nd International Conference on. IEEE, 2005.

[2] Cheng, Xiuzhen, et al. "Relay sensor placement in wireless sensor networks," Wireless Networks 14.3 (2008): 347355.

[3] Poduri, Sameera, et al. "Sensor network configuration and the curse of dimensionality," Proc. Third Workshop on Embedded Networked Sensors (EmNets 2006), Cambridge, MA, USA. 2006.

[4] Xu, Kenan, et al. "Optimal wireless sensor networks (WSNs) deployment: minimum cost with lifetime constraint," Wireless And Mobile Computing, Networking And Communications, 2005.(WiMob'2005), IEEE International Conference on. Vol. 3. IEEE, 2005.

[5] Chu-Fu Wang, Jen-Wen Ding, "The optimum sensor redeployment scheme using the most frangible clusters set," Computer Communications, Volume 31, Issue 14, 5 September 2008, Pages 3492-3502, ISSN 0140-3664, 10.1016/j.comcom.2008.06.004.
[6] Zhao Cheng, Mark Perillo, Wendi B. Heinzelman, "General Network Lifetime and Cost Models for Evaluating Sensor Network Deployment Strategies," IEEE Transactions on Mobile Computing, vol. 7, no. 4, pp. 484-497, April, 2008

[7] Feng Wang, Dan Wang, and Jiangchuan Liu " TrafficAware Relay Node Deployment: Maximizing Lifetime for Data Collection Wireless Sensor Networks," IEEE Trans. Parallel Distrib. Syst. 22, 8 (August 2011), 1415-1423.

[8] Wang, Quanhong, et al. "On relay node placement and locally optimal traffic allocation in heterogeneous wireless sensor networks," Local Computer Networks, 2005. 30th Anniversary. The IEEE Conference on. IEEE, 2005.

[9] Wang, Quanhong, et al. "Minimum cost guaranteed lifetime design for heterogeneous wireless sensor networks (WSNs)," Performance, Computing, and Communications Conference, 2005. IPCCC 2005. 24th IEEE International. IEEE, 2005.

[10] Wang, Quanhong, et al. "Locally optimal relay node placement in heterogeneous wireless sensor networks," Global Telecommunications Conference, 2005. GLOBECOM'05. IEEE. Vol. 6. IEEE, 2005.

[11] Younis, Mohamed, and Kemal Akkaya. "Strategies and techniques for node placement in wireless sensor networks: A survey," Ad Hoc Networks 6.4 (2008): 621-655.

[12] Heinzelman, Wendi Rabiner, Anantha Chandrakasan, and Hari Balakrishnan. 'Energy-efficient communication protocol for wireless microsensor networks," System Sciences, 2000. Proceedings of the 33rd Annual Hawaii International Conference on. IEEE, 2000.

[13] Lindsey, Stephanie, and Cauligi S. Raghavendra. "PEGASIS: Power-efficient gathering in sensor information systems," Aerospace conference proceedings, 2002. IEEE. Vol. 3. IEEE, 2002.

[14] Manjeshwar, Arati, and Dharma P. Agrawal. "TEEN: a routing protocol for enhanced efficiency in wireless sensor networks," 1st International Workshop on Parallel and Distributed Computing Issues in Wireless Networks and Mobile Computing. Vol. 22. 2001.

[15] Lindsay, Stehpanie, Cauligi S. Raghavendra, and Krishna M. Sivalingam. "Data gathering in sensor networks using the energy delay metric," Proceedings of the 15th International Parallel \& Distributed Processing Symposium. IEEE Computer Society, 2001.

[16] Haitao Zhang, Cuiping Liu. "A Review on Node Deployment of Wireless Sensor Network,"IJCSI International Journal of Computer Science Issues. Vol . 9. 2012.

[17] Paul Smith, Jo Agila Bitsch Link, Klaus Wehrle.'Demo: Opportunistic Deployment Support for Wireless Sensor Networks,"ExtremeCom 2013. 